\title{
Generation of inner ear sensory neurons using blastocyst complementation in a Neurog $1^{+/-}$-deficient mouse
}

\author{
Aleta R. Steevens ${ }^{1,2,3^{*}}$ D, Matthew W. Griesbach ${ }^{4}$, Yun You ${ }^{5}$, James R. Dutton ${ }^{3}$, Walter C. Low ${ }^{2,3}$ and Peter A. Santi ${ }^{3,4}$
}

\begin{abstract}
This research is the first to produce induced pluripotent stem cell-derived inner ear sensory neurons in the Neurog $1^{+/-}$heterozygote mouse using blastocyst complementation. Additionally, this approach corrected nonsensory deficits associated with Neurog 1 heterozygosity, indicating that complementation is specific to endogenous Neurog 1 function. This work validates the use of blastocyst complementation as a tool to create novel insight into the function of developmental genes and highlights blastocyst complementation as a potential platform for generating chimeric inner ear cell types that can be transplanted into damaged inner ears to improve hearing.
\end{abstract}

Keywords: Inner ear, Spiral ganglion neurons, Neurogenin1, Blastocyst complementation, Stem cells, Regenerative medicine

\section{Introduction}

Hearing loss is the most common neurosensory deficit. Approximately 488 million individuals world-wide and $15 \%$ of Americans have some degree of hearing loss [1]. Hearing depends on the mechano-sensory hair cells (HCs) and their innervating neurons, the spiral ganglion neurons (SGNs), which are responsible for transmitting auditory information from the HCs in the organ of Corti to the cochlear nucleus in the brainstem. Mammalian HCs and SGNs do not regenerate after damage, which results in sensorineural hearing loss (SNHL) [2]. In addition, auditory neuropathy with relative preservation of hair cells is a substantial cause of deafness [3, 4]. Cochlear implants are the only established therapy for severe to profound hearing loss; however, they require a viable SGN population for their success and efficacy [5].

\footnotetext{
* Correspondence: asteeven@umn.edu

'Department of Ophthalmology, University of Minnesota, Minneapolis, MN, USA

${ }^{2}$ Department of Neurosurgery, University of Minnesota, Minneapolis, MN, USA

Full list of author information is available at the end of the article
}

Using exogenous stem cells to replace lost inner ear neurons is a potential strategy if stem cell-derived neurons can form central and peripheral connections, form synapses on hair cells and cochlear nucleus neurons, and re-establish functional and tonotopic circuits [6]. While, early attempts to target cochlear tissues using stem cells largely produced unremarkable results [6,7], two promising in vivo studies have shown that stem cells can survive and supplement SGNs within a cochlea and even partially restore hearing function $[8,9]$. However, there are two potential weakness in the previous studies: (1) since the transplanted stem cells were differentiated in vitro to form otic progenitors and due to the lack of using established markers of inner ear specific neurons, these cells may not be equivalent to SGNs formed in vivo, which may explain the observed limited functional recovery, and (2) using allogenic stem cells requires that the transplant recipient may need to undergo long-term immunosuppression to prevent rejection of the transplanted progenitors. We address these potential limitations by adopting the technique of blastocyst 
complementation $(\mathrm{BC})$ to generate inner ear neurons from induced pluripotent stem cells (iPSCs).

$\mathrm{BC}$ is a technique in which deletion of a key gene for the development of a specific lineage creates a vacant niche (organogenesis-disabled phenotype) that can be complemented by the progeny of wild type pluripotent stem cells injected into embryos at the blastocyst stage of development. Resulting chimeras from BC have successfully generated entire, functional organs, such as the pancreas, kidney, eye, and lung, derived from the progeny of donor stem cells, by complementing genes necessary for their formation (pancreatic and duodenal homeobox 1 (PDX1), Sal-like like protein 1 (SALL1), melanocyte inducing transcription factor (MITF), and fibroblast growth factor receptor 2 (FGFR2), respectively [10-15].

These studies, using both intraspecies (mouse $\mathrm{X}$ mouse [11, 14] and pig X pig [12]) and interspecies (rat $X$ mouse $[10,13])$ chimeras, demonstrated the feasibility of $\mathrm{BC}$ to generate organs. Organogenesis is a complex developmental process requiring hierarchical cell and tissue differentiation, coordinated in time and space in response to changes in local and distant signaling cues. Replicating these conditions in vitro to generate functional tissues, let alone the organs, has proven extremely challenging and using the embryo to initiate the appropriate signaling cascades is a significant advantage of a $\mathrm{BC}$ approach. $\mathrm{BC}$ is the only current method for making fully functional, three-dimensional organs from pluripotent cells and generating human organs in large mammalian hosts may be able to address the critical worldwide problem of organ shortages for transplantation [16]. However, several hurdles still need to be overcome to make interspecies organ farming a reality [17]. For one, all components of an organ will need to be derived from the human tissue, which includes the endothelial, stromal, and possibly the immune cells [18-20]. While progress has been made on this front [21], it will take time to advance to point of being able to generate entirely the whole organs with $\mathrm{BC}$.

By contrast, the replacement of specific cell types could improve health conditions such as pancreatic beta islet cells for type 1 diabetes [22, 23], dopaminesecreting neurons in Parkinson's disease (PD) [24], and inner ear sensory cells for hearing disorders and may be a more readily attainable goal. $\mathrm{BC}$ has been used to generate rat islet cells that normalized glucose levels after transplantation into diabetic mice [25], and candidate genes have been identified to create replacement dopaminergic neurons using $\mathrm{BC}$ for PD [16]; however, $\mathrm{BC}$ has not been used to generate sensory cell types in the inner ear. Here, we present the first demonstration using intraspecies $\mathrm{BC}$ to create mouse donor stem cell-derived inner ear sensory neurons.
Ma and colleagues [26, 27] developed a Neurogenin1

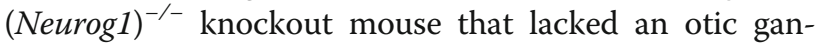
glion at embryonic day (E)10.5, which translated into postnatal day (P)0 inner ears that lacked afferent, efferent, and autonomic nerve fibers. In preliminary experiments using the Neurog $1^{-/-}$mutant mouse, we produced the first histological analysis of E18.5 Neurog $1^{-/-}$null inner ears [28], which confirmed the findings of $\mathrm{Ma}$ and colleagues. With the goal of generating stem cell-derived inner ear neurons, the Neurog $1^{-/-}$knockout animal was the optimal choice for creating a vacant niche to generate inner ear neurons by blastocyst complementation.

\section{Methods}

All methods performed in this manuscript were in accordance with all policies of the University of Minnesota Institute of Animal Care Use Committee (IACUC), which approved the use and housing of these animals according to accepted principles of laboratory animal care (National Research Council 2003).

\section{Mice}

The Neurog1 $1^{\text {tm1And/J }}$ mouse strain was used [26, 27] (Jackson Laboratory, Stock No. 017306) in which the coding exon for Neurog1 was replaced with a green fluorescent protein (GFP) cassette fused to a PGK-neo cassette successfully abolishing gene function in $\mathrm{Neu}$ rog $1^{-/-}$null mice [26-28]. Expression of the GFP mRNA was reported to mirror Neurog1 endogenous gene function which confirmed the specificity of the transgene knock in. However, GFP protein expression was undetectable indicating that the GFP transcript generated in these mice results in a nonfunctional GFP protein [26]. Mice were housed in a specific pathogen-free (SPF), Research Animal Resource (RAR) and American Association for Accreditation of Laboratory Animal Care, (AAALAC) approved facility, and plastic cages that were steam cleaned and autoclaved 3 times per week. The mouse colony was maintained by crossing $\mathrm{Neu}$ rog $1^{+/+}$wildtype mice with Neurog $1^{+/}$heterozygous mice. Experimental mice were generated through heterozygous matings (Neurog1 ${ }^{+/-}$X Neurog $1^{+/-}$), which produced the following combinations of genotype: $\mathrm{Neu}$ rog $1^{+/+}$wild-type, Neurog $1^{+/-}$heterozygous, Neurog $1^{-/-}$ null mice. Neurog $1^{-/-}$null mice die $24 \mathrm{~h}$ after birth due to their inability to suckle and were only generated for blastocyst complementation or embryonic harvest. Toe clips were collected at 1 week of age, which were subsequently hydrolyzed for genotyping analysis. Genotyping was performed by polymerase chain reaction (PCR) using the Jackson Laboratory's validated genotyping protocol: https://www.jax.org/Protocol?stockNumber $=01$ 7306\&protocolID $=23926$. 
Genotyping was performed with the following primers: WT forward (ACCACTAGGCCTTTGTAAGG), mutant forward (ATAGACCGAGGGCAGCTTCA), and a common reverse primer (CGCTTCCTCGTGCTTTACGG TAT). Genotyping was run in two separate reactions: (1) WT forward, a sequence which detects endogenous Neurog1 protein, and the common primer and (2) the mutant forward, which is a sequence in the neomycin cassette, and the Common primer. This reaction yields a 198-bp wild-type band and a 500-bp mutant band. Wild-type animals will have only one wild-type band, heterozygotes will have both wild type and mutant bands, and homozygous nulls will only have a mutant band (Supplemental Figure 1).

\section{Blastocyst complementation}

Mouse $\mathrm{x}$ mouse blastocyst complementation was performed by injecting membrane-bound GFP-labeled mouse iPSCs into Neurog1-deficient blastocysts. The derivation of the 3F10 iPSC line used was previously described in detail by Greder and colleagues [29]. Briefly, the iPSCs were derived from Oct4-MerCreMer mTmG mice. These mice are from a cross between Oct4MerCreMer mice carrying a tamoxifen-inducible Cre knock-in transgene upstream of the 3'UTR of Oct4 (Jackson Laboratory, Stock No: 016829) and a homozygous tdTomato/EGFP reporter mouse strain (mTmG) (Jackson Laboratory, Stock No. 007576). iPSCs were generated according to previous reprogramming protocols $[30,31]$ and resulting pluripotent iPSCs were cultured with tamoxifen diluted in miPSC growth medium to $100 \mathrm{nM}$. This strategy results in all donor iPSCs being irreversibly labeled with membrane-bound EGFP (Supplemental Figure 2). Injected blastocysts were transferred to pseudopregnant female surrogates, where they were allowed to develop until the time of natural birth. To produce mutant blastocysts, an in vitro approach was used. To do this, the egg donors (Neurog $1^{+/-}$heterozygous female mice) were superovulated [32] at 3-4 weeks of age, by giving CARD HyperOva ${ }^{\bullet}$ (Cosmo Bio, Cat. No. KYD-010-EX-X5) $0.1 \mathrm{ml} /$ mouse, intraperitoneally (i.p.), at 17:30 pm (mouse room light:dark cycle: 6:00-20: 00), followed by human chorionic gonadotropin (HCG) (Sigma-Aldrich Cat. No. C 1063), 5 insulin units (IU)/ mouse $47-48 \mathrm{~h}$ later. Fresh sperm from a Neurog1 heterozygous (+/-) male was used for IVF. Fertilized eggs were cultured in home-made modified human tubal fluid (mHTF, a.k.a. high calcium HTF) medium until the blastocysts were formed in $\sim 72 \mathrm{~h}$ after IVF and ready for microinjection of iPSCs. Each blastocyst was injected with 10-15 iPSCs. After blastocyst injection, mouse blastocysts were transferred into the uteri of pseudopregnant surrogate mice. This entire process was performed on three separate occasions. Variability is expected with embryological development and some of the surrogate dams resorbed all the developing embryos. However, we obtained two viable litters and 13 P0 pups were obtained: 2 Neurog $1^{+/+}$wild type animals, 9 Neurog $1^{+/-}$ heterozygote animals, and 2 Neurog $1^{-/-}$homozygote null animals. Of these, one of the wild type samples was highly chimeric, three of the heterozygotes were complemented (30\%), and none of the homozygotes exhibited any stem cell-derived chimerism.

\section{Inner ear harvesting from the embryo, cryosectioning, and immunohistochemistry}

For tissue harvesting, P1 mice were euthanized using $\mathrm{CO}_{2}$, according to RAR guidelines, followed by decapitation. The heads were bisected with the intention of using one inner ear for cryosectioning and immunohistochemistry (IHC) and the other processed for imaging using scanning Thin Sheet Laser Image Microscope (sTSLIM). The bisected heads were fixed overnight in $4 \%$ paraformaldehyde (PFA) in phosphate-buffered saline (PBS) and were rinsed with PBS twice for $15 \mathrm{~min}$ each before undergoing decalcification in $10 \%$ ethylenediaminetetraacetic acid (EDTA) for 3 days. Inner ears to be analyzed by cryosectioning and $\mathrm{IHC}$ were cryoprotected through overnight incubations in ascending concentrations of sucrose up to $30 \%$ and embedded in tissue-freezing medium (TFM) (General Data, Cat\#: TFM-5) and snapfrozen on dry ice. Bisected heads containing the P0 inner ears were sectioned on a cryostat at a thickness of $16 \mu \mathrm{m}$ and directly mounted to slides. The entire ear was collected, starting with sections from the cochlea, through the macular organs, and through the cristae and semicircular canals. Slides were allowed to dry on the slide warmer for a minimum of $1 \mathrm{~h}$ prior to beginning staining or storing at $-20^{\circ} \mathrm{C}$. The sectioned tissue was always stained within 3 days of sectioning. If previously frozen, the tissue was rewarmed to room temp by placing it on the slide warmer for a minimum of a half hour. Prior to performing immunohistochemistry, epitopes were exposed by performing antigen retrieval. Briefly, slides were placed in Coplin jars filled with boiling sodium citrate buffer with Tween ${ }^{\odot} 20$ (Sigma-Aldrich Cat\# P9416) ( $\mathrm{pH}$ adjusted to 6.0 with $1 \mathrm{~N}$ hydrogen chloride $(\mathrm{HCl})$ ) and incubated in a steamer for $20 \mathrm{~min}$. Slides were allowed to cool to room temperature, for at least an hour, before continuing the staining protocol. After antigen retrieval, the slides were dried and the sections were outlined with a hydrophobic barrier pen $\left(\right.$ Super $^{\mathrm{HT}}$ Pap Pen, Polysciences Cat\# 24230-1). The tissue was rinsed twice (10 min each rinse) with PBS and rinsed twice (15 min each rinse) in PBS with $0.1 \%$ Triton $^{\mathrm{Tm}} \mathrm{X}-100$ (Sigma-Aldrich, X100, Cat\# 9002-93-1) (PBST). Nonspecific binding was blocked against using 10\% normal horse serum (ThermoFisher, Cat\# 16050122) in 0.1\% 
PBST for $1 \mathrm{~h}$ at room temperature. Primary antibodies, diluted in the blocking solution, were applied and allowed to incubate in the $4{ }^{\circ} \mathrm{C}$ cold room overnight. All antibodies are listed in the below table (Tables 1 and 2). The following day, the slides were rinsed with four 15min washes in $0.1 \%$ PBST, and sections were incubated in secondary antibodies, diluted in blocking solution, for $2 \mathrm{~h}$ at room temperature. The tissue was then rinsed in $0.1 \%$ PBST with two 10-min washes and counterstained with 4',6-diamidino-2-phenylindole (DAPI, Thermo Fisher Scientific, Cat\# D1306) at a concentration of 1: 5000 for $5 \mathrm{~min}$. Lastly, the tissue was rinsed for $10 \mathrm{~min}$ in PBS, and coverslips were mounted to the slide after applying the mounting medium with anti-fade agent (Electron Microscopy Services, Cat\# 17985-11). Slides were sealed using CoverGrip $^{\text {тм }}$ Coverslip Sealant (Thermo Fischer Scientific, Cat\# NC0154994).

\section{Microscopy and image processing}

All immunohistochemical imaging was performed on a Leica inverted light microscope. Images were exported as raw Leica Image File Format (LIF) files and processed in FIJI (Fiji Is Just ImageJ). The resolution of each image was adjusted to 300 dots per inch, which reflects pixels per inch (DPI) in Photoshop, and all resulting JPEGs were assembled using Adobe Illustrator.

\section{sTSLIM macro light-sheet microscope}

In 2008, we developed a high-resolution microtome/ microscope called scanning Thin Sheet Laser Image Microscope (sTSLIM) that can image whole cochleas, nondestructively [33-37]. sTSLIM optically sections and digitizes all cochlear tissues to allow for a complete quantitative assessment of normal and pathological structures. The Santi laboratory has used STSLIM to characterize mouse cochlear development [38], analyze normal spiral ganglion cell number in the mouse [39], and illustrate alterations in cochlear structures in two knock-out mouse models (Atonal homolog1, ATOH1 and $N$-Myc proto-oncogene protein, $N$-Myc) [40, 41]. Since light scatter and absorption are the greatest limiting factors in resolution, we have performed both tissue engineering (improved transparency and accessibility to antibody labeling through decellularization) and optical engineering (scanned light-sheet, Bessel beam illumination, structured illumination, confocal line detection, and radial sectioning) to improve imaging of large specimens such as the mouse cochlea with portions of the brain attached. Microscope performance will be tested on a regular basis using 150-nm gold fluorescent beads to ensure that the point spread function of the microscope is stable and optimal for reproducible imaging.

\section{Tissue preparation for STSLIM}

Inner ears processed for sTSLIM were fixed and decalcified (described above) and then underwent a dehydration series in ascending concentrations of ethanol $(30 \%$, $50 \%, 70 \%, 100 \% \mathrm{EtOH})$ and then lightly stained by rhodamine-B isothiocyante $(5 \mu \mathrm{g} / \mathrm{mL}$ in $100 \%)$ for $1 \mathrm{~h}$. After rinsing in $100 \% \mathrm{EtOH}$, inner ears were cleared to transparency with BABB (benzyl alcohol: benzyl benzoate 1:2), and specimens were mounted to a specimen rod and placed in a BABB-filled chamber for imaging by sTSLIM.

\section{sTSLIM imaging}

sTSLIM optically sections non-destructively by moving a thin light sheet in the $x$ - and $Z$-axes. A $z$-stack of wellaligned 2D optical sections of the inner ear was automatically imaged with $x$-axis scanning across the width of the specimen and with a $z$-step size of $5 \mu \mathrm{m}$. At this thickness, the whole mouse cochleae contained $\sim 300$ images that take $\sim 30 \mathrm{~min}$ to produce. Images were adjusted for brightness, contrast, and either unsharp masking or deconvolution using ImageJ (National Institutes of Health). The $z$-stack was then loaded into the Amira 3D program, and structures of interest were manually segmented, by drawing along their borders in different colors to prepare 3D reconstructions and volume calculations. Supplemental Figure 3 shows an example of a 2D optical section through the cochlea and Supplemental Movie 1 shows segmented inner ears rotating horizontally.

\section{Results \\ Donor GFP-labeled stem cells create chimeric spiral ganglion and vestibular neurons in the Neurog $1^{+/-}$ heterozygote}

Neurog1-deficient blastocysts were established by performing in vitro fertilization (IVF) with zygotes extracted from Neurog $1^{+/-}$heterozygous dams and fresh sperm from Neurogl $^{+/-}$heterozygous male mice. Blastocysts were injected with EGFP-labeled mouse induced

Table 1 Antibodies

\begin{tabular}{llll}
\hline Antibody & Vendor & Cat\# & Dilution \\
\hline rabbit polyclonal a-MYO6 & Proteus Biosciences Inc. & $25-6791$ & $1: 500$ \\
chicken a-GFP & Abcam Inc. & ab13970 & $1: 1000$ \\
mouse monoclonal a-Tuj1 & Biolegend & MMS-435P & $1: 1000$ \\
\hline
\end{tabular}


Table 2 Antibodies

\begin{tabular}{llll}
\hline Antibody & Vendor & Cat\# & Dilution \\
\hline 488 donkey a-chicken & Jackson ImmunoResearch Alexa Fluor & $703-545-155$ & $1: 1000$ \\
555 donkey a-rabbit & Invitrogen Alexa Fluor & Ab150062 & $1: 1000$ \\
647 donkey a-mouse & Invitrogen Alexa Fluor & A-31571 & $1: 1000$ \\
\hline
\end{tabular}

pluripotent stem cells (iPSCs) at approximately embryonic day (E) 3.5 and subsequently were transferred into surrogate pseudopregnant dams (Fig. 1a). The iPSCs were derived from transgenic mice that carried a tamoxifen-inducible CreER transgene under the control of the Oct4 promoter and a Cre-dependent tdTomato/ EGFP reporter cassette. The iPSCs used in this study were from cells previously cultured with tamoxifen to activate the Oct4 driven CreER, removing the tdTomato reporter gene and activating EGFP expression. As Oct4 is highly expressed in iPSCs, this strategy irreversibly labels all the donor stem cells and their progeny with EGFP (Supplemental Figure 2). Upon analysis of chimeric inner ears at P1, robust and specific incorporation of GFP-labeled cells derived from the donor stem cells was seen in the SGN of a Neurog $1^{+/-}$heterozygote (Fig. 1).

In the complemented Neurog1 ${ }^{+/}$, GFP-labeled iPSCs contributed to the SGN and descending neuronal processes in the cochlea with minimal donor stem cellderived cell incorporation in other tissue (Fig. 1d, e arrowheads). The specificity of labeling in the Neurog1 ${ }^{+-}$ was unmistakable, given the lack of GFP labeling observed in the wild type SGN (Fig. 1c, f, arrows). Progeny of the GFP-labeled donor iPSCs also contributed to the cell bodies of Scarpa's ganglion (Fig. 1g), in addition to vestibular neurons innervating the cristae ampullaris (Fig. 1h, i). Since Neurog1 is known to be required for the formation of both cochlear and vestibular neurons [27], these results indicate that the integration pattern of cells derived from the wild type donor cells recapitulates that expected from cells with wild type endogenous $\mathrm{Neu}$ rog1 gene function and that Neurog1 haplodeficiency creates a vacant niche that can be filled by cells derived from exogenous stem cells to produce SGNs using BC.

\section{Complementation of Neurog1-deficiency is distinct from general chimerism}

A chimera is defined as a composite animal comprised of two genetically distinct cell populations [42]. Performing $B C$ in wild type animals will result in random chimerization throughout the developing embryo [43, 44]. When performing $\mathrm{BC}$ in knockout blastocysts, in addition to exogenous donor-derived cells targeting a vacant developmental niche, random chimerism can also be observed in non-targeted cell types.
Two examples of complemented Neurog1 ${ }^{+/-}$heterozygotes were highly chimeric, as a high degree of specific donor-derived cell integration into the SGN was observed (Fig. 2b-D'), in addition to general chimerism, which was evident from the widespread GFP expression in non-sensory otic cell types (Fig. 2b-D'). This general chimerism is similar to that observed in the Neurog $1^{+/+}$ control, which also displayed the incorporation of GFPexpressing cells in non-sensory cells. Notably, the $\mathrm{Neu}$ rog $1^{+/-}$heterozygote SGNs, in some regions, appeared to be derived nearly entirely from donor iPSCs, whereas the Neurog ${ }^{+/+}$wild type SGNs were consistently negative for GFP expression (Figs. 1c and 2a, A').

The extent of donor-derived cell chimerism in the Neurog $1^{+/+}$wild type in comparison to the complemented Neurog $1^{+/-}$heterozygotes was assessed further by looking at the respective level of GFP expression in the temporal lobe of the brain. This analysis clearly showed the incorporation of exogenous iPSC-derived cells in the Neurog $1^{+/+}$brain at a comparable level to the complemented Neurog $1^{+/-}$heterozygotes (Fig. 2e, f). Therefore, chimeras successfully formed regardless of genotype, but in the absence of a Neurog1-deficient niche, GFPexpressing cells derived from the exogenous donor iPSCs could not contribute to the formation of the SGN in wild type inner ears (Figs. 1f and 2Aa'). These results support our hypothesis by demonstrating that $\mathrm{BC}$ can specifically generate SGNs using WT donor stem cells.

We did not see complementation in the two $\mathrm{Neu}$ $\operatorname{rog}^{-/-}$mutant embryos that we obtained. However, both nulls showed few to no GFP-expressing cells engrafted throughout the whole embryo, suggesting that the lack of complementation was due to low chimerism. With an increased sample size, we expect to obtain highly chimeric Neurog $1^{-/-}$mutants in which all of the SGNs are derived from donor stem cell progeny. Therefore, it is anticipated that, following Mendel's law, 75\% of blastocysts (heterozygous and null) obtained from BC will give rise to chimeric inner ears.

\section{Donor stem cells extensively contribute to the complemented Neurog $1^{+/-}$vestibule}

In the highly chimeric complemented Neurog ${ }^{+/-}$inner ears, the contribution of donor-derived GFP-labeled cells appeared to increase in sections through the more basal cochlea in a trend that was dramatically more evident in the vestibule. In fact, it appeared that the majority of 


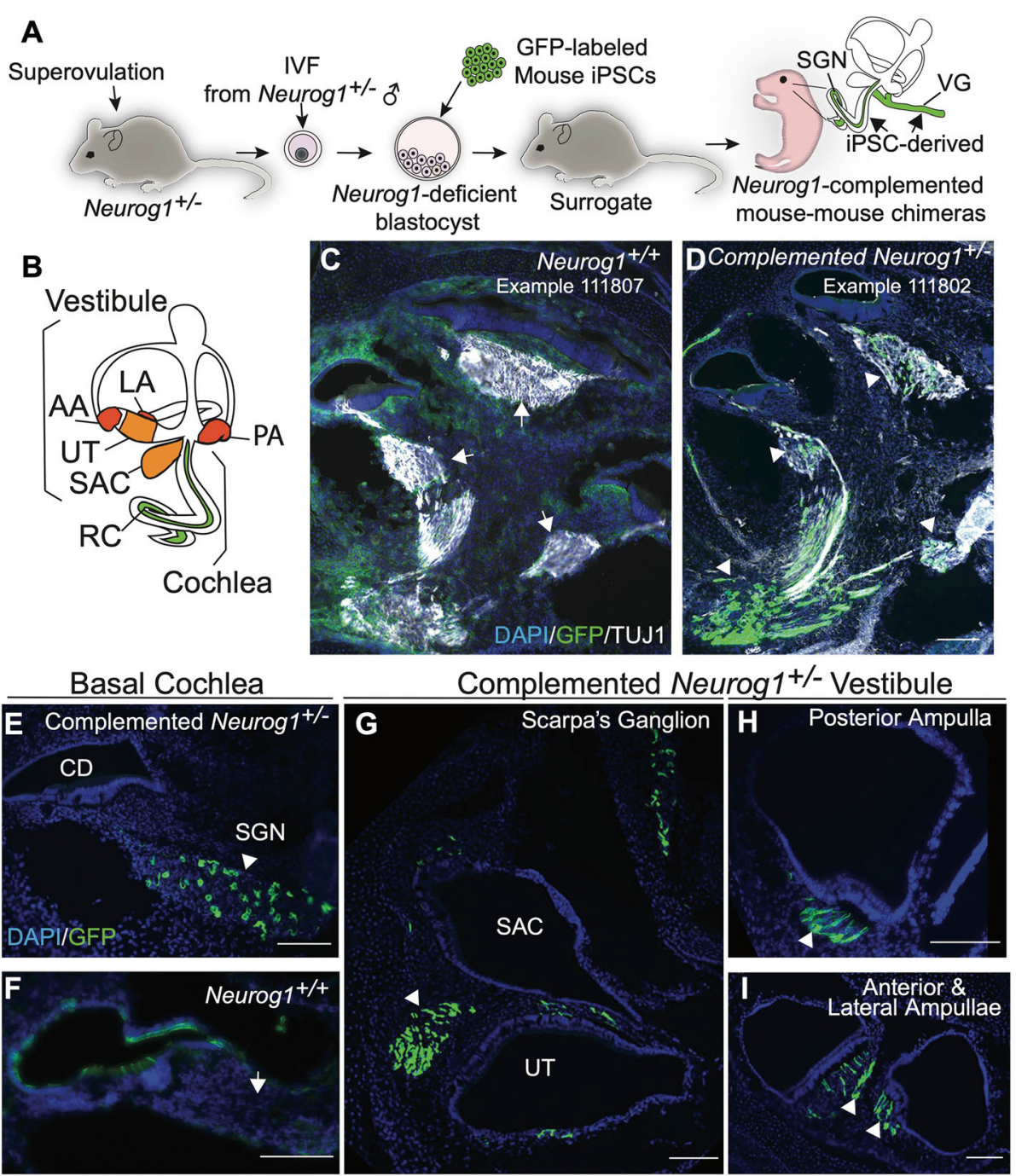

Fig. 1 Donor GFP-labeled stem cells create chimeric spiral ganglion and vestibular neurons in the Neurogl heterozygote. a Schematic of experimental methods for BC. Neurog $1^{+/-}$female mice were superoverulated with hormones. Zygotes were extracted and fertilized by in vitro fertilization (IVF) with Neurog $1^{+/-}$male sperm and allowed to mature until blastocyst stage ( E3.5), at which point all blastocysts were injected with GFP-labeled iPSCs. Complemented blastocysts were transferred to a pseudopregnant surrogate. Pregnancies were to taken to birth, embryos were harvested, and complementation in the inner ear was assessed. b Diagram of the inner ear indicates the vestibular (balance) portion and cochlear (auditory) portion. Low magnification of the midmodiolar region of the cochlea in $\mathbf{c}$ representative image of the Neurog $1^{+/+}$wild type inner ear shows the absence of GFP-labeled stem cells in the SGN (arrows) (images are from a single Neurog $1^{+/+}$wild type (1/2), sample number 111807) in contrast to $\mathbf{d}$ specific stem cell complementation in the SGN in a Neurog $1^{+/-}$heterozygote (arrowheads) (images are from a single complemented Neurog $1^{+/-}$heterozygote (1/3), sample number 111802). High-magnification images of the basal cochlear turn show e the presence of GFP-labeled stem cells in a Neurog $1^{+/-}$heterozygote SGN (arrowhead) and absence in $\mathbf{f}$ the Neurog $1^{+/+}$wild type SGN (arrow). Specific stem cell complementation was seen in the vestibule of a Neurog $1^{+/-}$heterozygote, $\mathbf{g}$ in Scarpa's ganglion adjacent to the utricle (UT) and saccule (Sac), $\mathbf{h}$ neurites (arrowhead) innervating the posterior ampulla, and $\mathbf{i}$ neurites innervating the anterior and lateral ampullae (arrowheads). AA anterior ampulla, LA lateral ampulla, PA posterior ampulla, Ut utricle, SAC saccule, RC Rosenthal's canal with SGNs. All scale bars are $100 \mu \mathrm{m}$. Scale bar in $\mathbf{d}$ also corresponds to $\mathbf{c}$

cells in the vestibule were derived from donor iPSCs, given the extensive presence of GFP in all vestibular cell types. Specifically, GFP entirely co-expressed with the neuronal marker class III beta-tubulin (TUJ1) in the neurites innervating the vestibular sensory organs (Fig. 3a-B', arrowhead and dotted lines), in addition to many Myosin 6 (MYO6)- expressing vestibular hair cells (Fig. 3a, b, red) and nonsensory cells (Fig. 3A', B', small arrows). While the degree of donor cell contribution to the complemented Neurog1+/ - vestibule was striking, the biological significance of this was lacking, until a clear heterozygote effect in noncomplemented Neurog ${ }^{+/-}$inner ears was detected. 


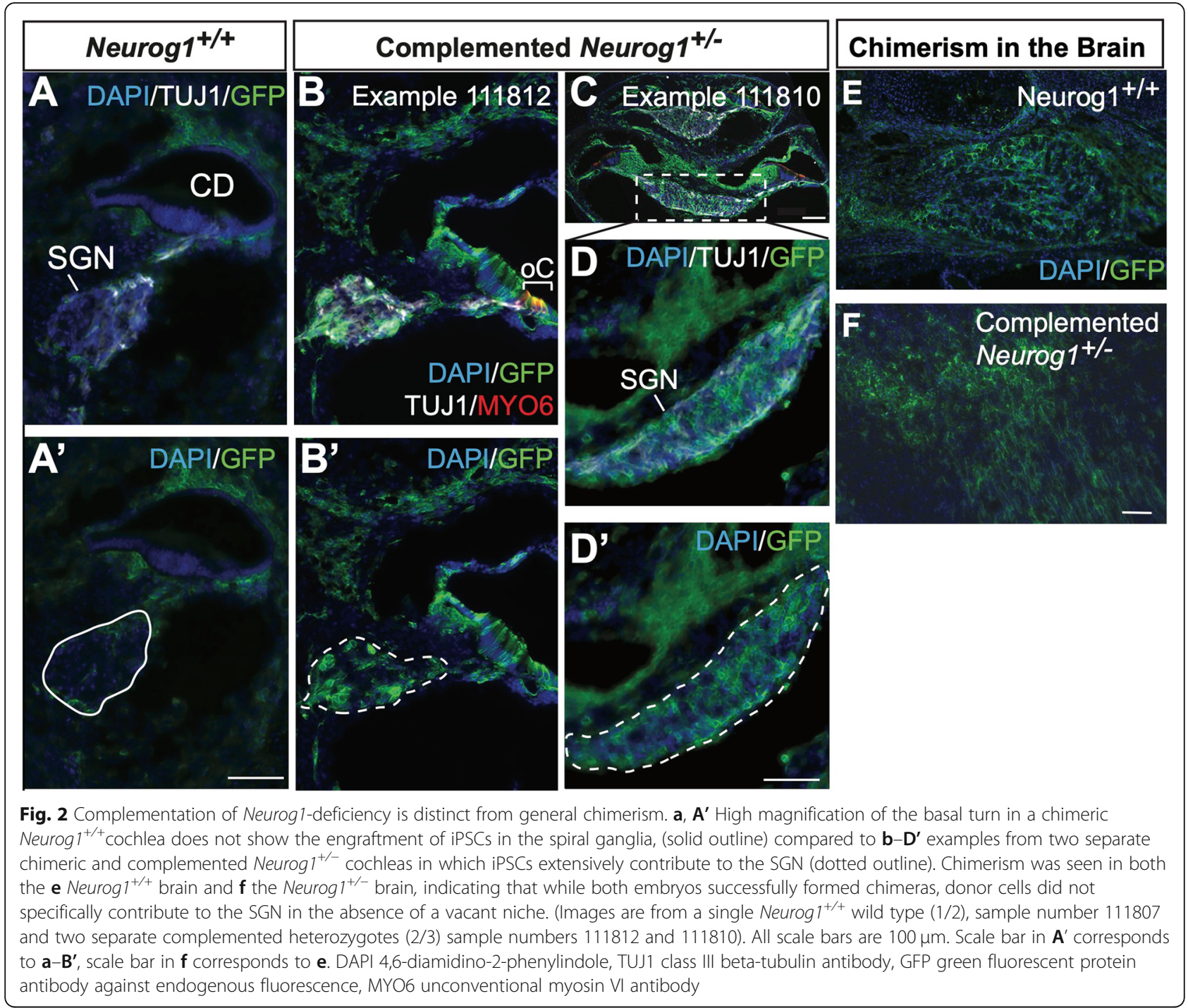

Blastocyst complementation rescues Neurog $1^{+/-}$inner ear malformations

Non-complemented Neurog $1^{+/-}$heterozygote inner ears were observed to have inner ear morphological nonsensory malformations, which included inner ears reduced in size by approximately $60 \%$ of the wildtype control (Fig. 4a, Supplemental Movie 1). Three-dimensional reconstructions revealed overt malformations particularly in the vestibule, in which the anterior and lateral ampullae and the saccule were notably reduced in size (Fig. 4a-d, Supplemental Movie 1). This finding was confirmed via cryosections, which displayed that the non-complemented Neurog $1^{+/-}$vestibule sometimes had an abnormally orientated saccule, utricle, and anterior ampullae. Specifically, unlike the typical orthogonal arrangement of the utricular and saccular maculae observed in the wild type vestibule, the non-complemented Neurog $1^{+/-}$sensory maculae (denoted by MYO6- expressing hair cells) were oriented in parallel (Fig. 4e, f; red, small arrows). Additionally, the utricular maculae and anterior crista ampullaris were unusually close to one another (Fig. 4f, arrowheads). Moreover, in some cases, the lateral ampulla appeared connected to the lateral semicircular canal (Fig. 4i, arrowheads). Impaired non-sensory vestibular morphology was observed in four of the six Neurog $1^{+/-}$heterozygotes obtained and analyzed. Strikingly, the remaining two Neurog $1^{+/-}$heterozygotes completely lacked a vestibule (not shown). Together these observations suggest that a reduction in non-sensory cell formation and/or a failure of sensory organ separation occurred with the reduction of Neurog1 gene dose.

Cochlear and vestibular HCs developed normally in the Neurog $1^{+/-}$heterozygote (as has been reported for the Neurog $1^{-1-}$ mutant) despite impaired non-sensory formation. However, sensory development potentially 


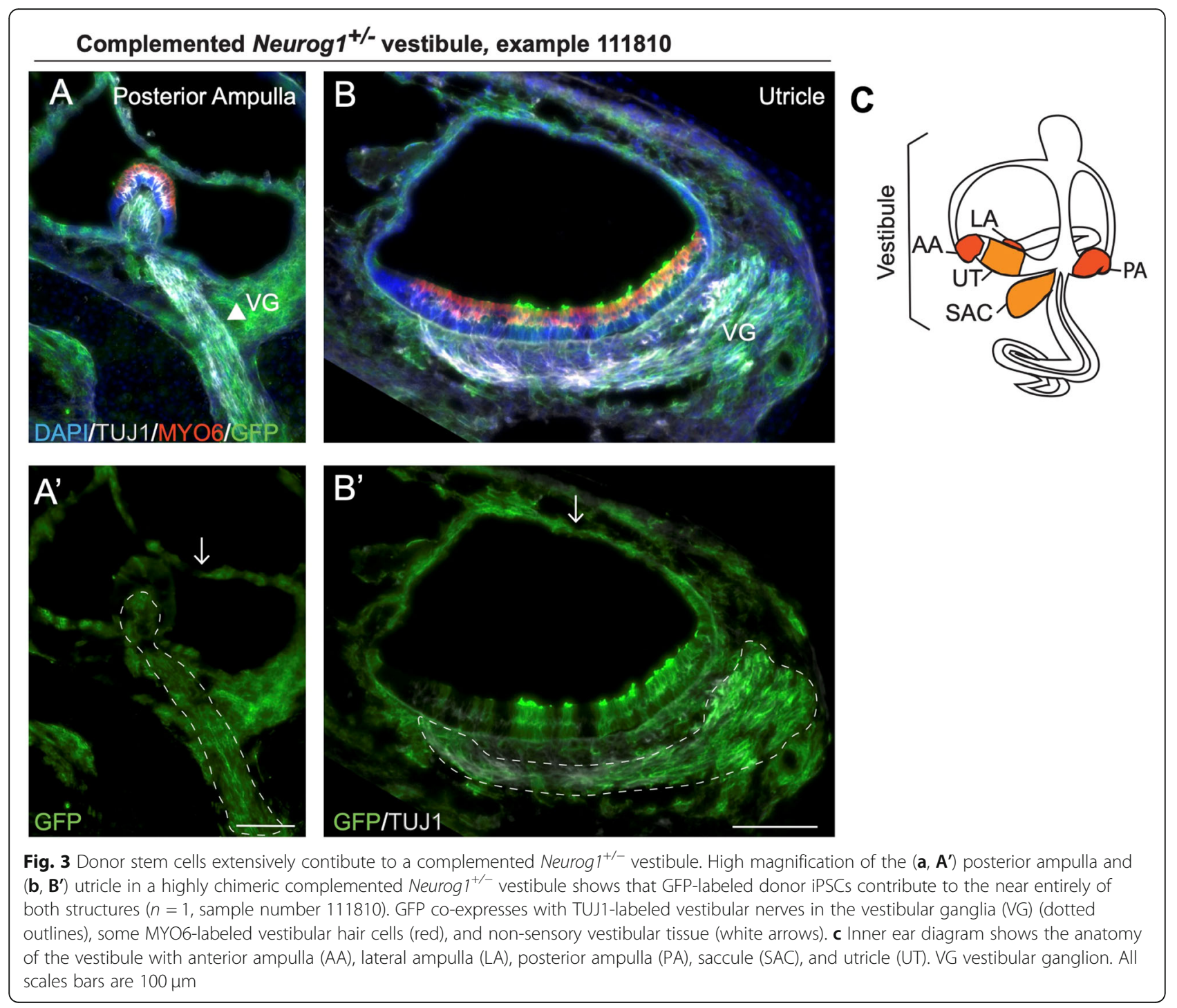

occurred at the expense of non-sensory development, as ectopic HCs were sometimes seen in non-sensory regions in the lateral semicircular canal (Fig. 4I').

Importantly, non-sensory defects observed in the Neu$\operatorname{rog}^{+/-}$heterozygotes (Fig. 4B, B', D, F, I) were rescued in complemented Neurog1 ${ }^{+/-}$samples (Fig. 4C, D, G, J). Given the extensive contribution of GFP to non-sensory tissue in the complemented Neurog $1^{+/-}$heterozygous vestibular sensory organs (Fig. 3), these results suggest that widespread incorporation of cells derived from the donor iPSCs to the vestibule in the complemented Neurog $1^{+-}$heterozygote is not random, but rather reflects the recovery of a previously unappreciated biological function of Neurog1 in inner ear morphogenesis. This finding demonstrates the use of $\mathrm{BC}$ as a tool to elucidate novel gene function and to confirm or disprove concepts regarding the development of neurobiological systems.

\section{Discussion}

Presented here for the first time is the intraspecies complementation of Neurog1-deficient mouse blastocysts to generate a chimeric population of donor iPSC-derived SGNs. This is a major advancement in the field of regenerative medicine for hearing disorders. The complexity of the intersecting developmental pathways involved in lineage specification of inner ear sensory cells is a difficult task to recapitulate in vitro [6]. By contrast, following blastocyst complementation, the developing embryo initiates these processes to produce what are presumed to be authentic spiral ganglion neurons. The approach of using BC to target Neurog1-deficiency is also advantageous, as Neurog1 is well-characterized to specify inner ear neuroblasts during the otocyst stage, a developmental stage when the future inner ear is a simple structure on a cellular level [45-47]. Therefore, it would be a straightforward workflow to (1) use blastocyst 


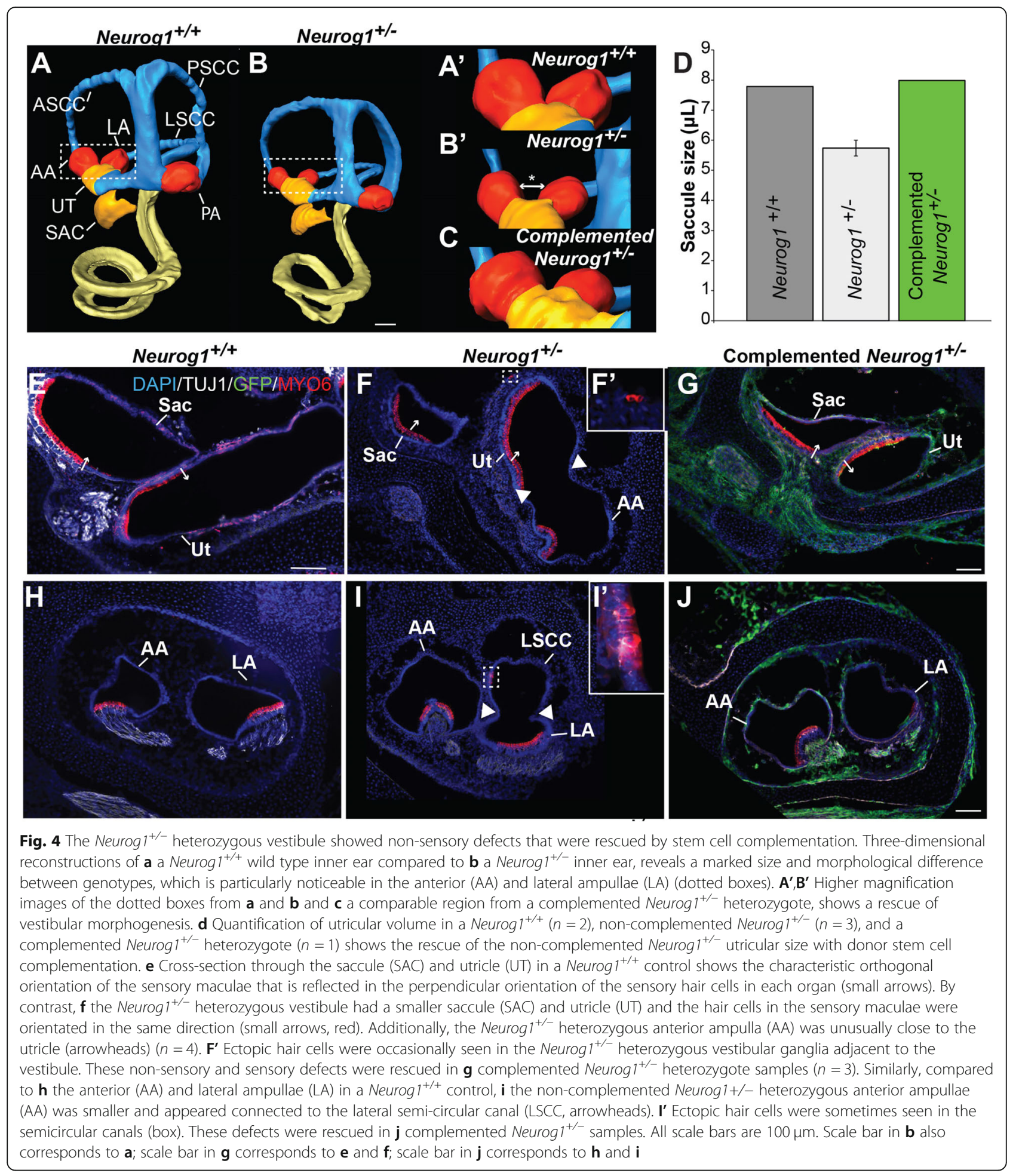

complementation to target a Neurog1-deficient niche in order to generate chimeric otocysts that can (2) be surgically extracted, (3) GFP-labeled neuroblasts derived from donor cells isolated by flow cytometry, and (4) could be transplanted to inner ears with damaged neuronal innervation. The prospect of isolating and transplanting autologous BC-derived cells has been successfully demonstrated when pancreatic islet cells were extracted from mouse-rat chimeras and transplanted into a chemically induced mouse diabetes model and normalized host blood glucose levels for over a year [25]. Moreover, developing the therapeutic application for using $\mathrm{BC}$ to 
create inner ear neurosensory cells is simpler than the goal of using $\mathrm{BC}$ for the generation of entire organs, which may require the nullification of several genes, such as those required for the development of the vasculature system [48] in order to prevent immune rejection.

Many outstanding hurdles exist in the $\mathrm{BC}$ field, which includes matching the developmental timing between the host blastocyst and donor cells of different species, which is a particular challenge for creating chimeras with human tissue. However, great strides have been made in better understanding the optimal cellular status in PSCs (naïve vs primed) necessary for successfully creating interspecies chimeras, in addition to ways of promoting survival of the donor cells [49-54]. Therefore, these barriers seem likely to be overcome, as indicated by the creation of successful human-animal chimeras $[55,56]$.

Using iPSCs for BC was designed to circumvent ethical issues associated with using embryonic stem cells (ESCs) and with the goal of creating autologous organs/tissues for transplantation to avoid immune rejection by the recipient. While a number of studies support this conclusion by showing that transplanted derivatives of pluripotent stem cells do not evoke an immune response $[57,58]$, a few recent studies have raised concerns [59] that include potential immunogenicity of iPSCs. Specifically, it has been found that human-induced pluripotent stem cells (hiPSCs) can sometimes be rejected by allogeneic and autologous natural killer cells (NK) [60] and cells derived from iPSCs can similarly activate the immune system [19]. Due to these mixed results, caution will be warranted when moving interspecies $\mathrm{BC}$ into the transplantation arena. However, safer alternatives to immunosuppressants are being developed. Since rejection is primarily mediated by $\mathrm{T}$ cell-dependent recognition of foreign antigens [61], strategies such as modulation of the $\mathrm{T}$ cell costimulatory and inhibitory pathways are being developed to safely protect the iPSC-derived transplants from immune rejection $[62,63]$. Other approaches to providing immune protection for transplants are by targeted disruption of the activation of antigen-presenting cells. For instance, blocking the expression of the major histocompatibility complex (MHC) class II transactivator in human embryonic stem cells (hESCs) leads to the silencing of HLA class II expression and thereby making these cells immune evasive. Therefore, there will likely be feasible options for developing safe and effective strategies to support iPSC-based regenerative medicine in the near future.

Unlike other studies using BC, we demonstrated that simply being haplodeficient for a master fatedetermining gene is sufficient to generate a $\mathrm{BC}$-derived tissue, which adds nuance to the complementation field. This unexpected finding led to the analysis of heterozygous inner ears with and without complementation and generated data that suggests that Neurog1 is dose- dependently required for non-sensory development while largely preserving neurosensory development. This may suggest that Neurog1 is involved in a binary decision to either adopt a non-sensory or neurosensory fate through an interaction with Notch signaling, a notion consistent with previous studies [26, 45, 46, 64]. Importantly, the observed non-sensory defects in the Neurog $1^{+/}$were rescued in successfully complemented chimeric samples (Fig. 4). This supports the idea that our complementation results cannot be attributable to general chimerism, but rather are specific to an endogenous role for Neurog1 in inner ear morphogenesis in addition to neurogenesis. Neurogl has been previously fate mapped in non-sensory regions of the cochlea and maculae [46]; therefore, this work provides evidence of a functional role for Neurog1 in these areas and supports the likelihood of Neurog1 playing additional roles in inner ear development beyond simply promoting inner ear neurogenesis.

Here, we establish, using intraspecies mouse chimeras, that $\mathrm{BC}$ is a platform that can be used for generating inner ear cell types. The generation of hiPSCs enables the generation of tissues that are either autologous or from closely related genetic background as the recipient [65]. Therefore, this work sets the stage for interspecies complementation with the goal of generating human inner ear tissues using swine as biological incubators, which could lead to improved research models and drug screening methodologies and ultimately transplantation to deaf patients to improve hearing.

\section{Supplementary Information}

The online version contains supplementary material available at https://doi. org/10.1186/s13287-021-02184-1

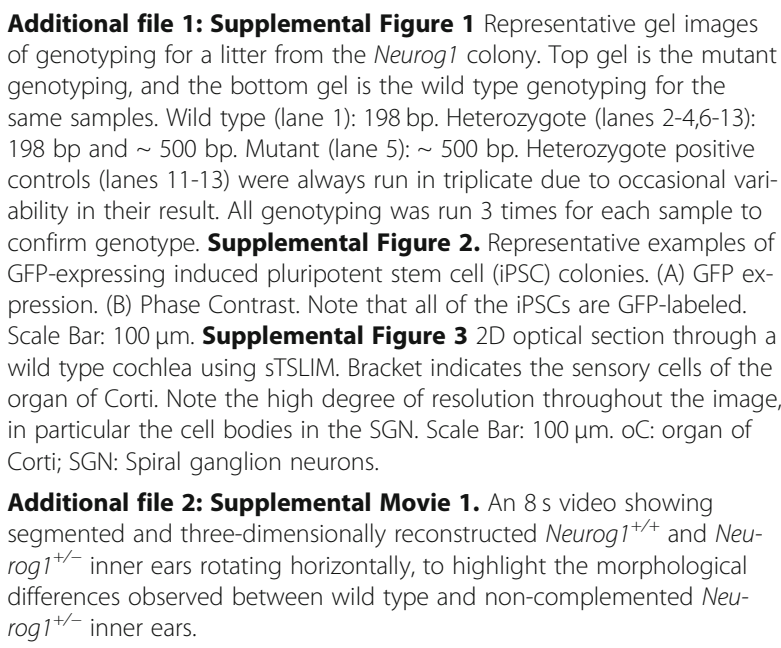

Additional file 2: Supplemental Movie 1. An $8 \mathrm{~s}$ video showing segmented and three-dimensionally reconstructed Neurog $1^{+/+}$and $\mathrm{Neu}$ rog $1^{+/-}$inner ears rotating horizontally, to highlight the morphological differences observed between wild type and non-complemented Neu$\operatorname{rog} 1^{+/-}$inner ears.

\section{Abbreviations}

AA: Anterior ampulla; AAALAC: American association for accreditation of laboratory animal care; ATOH1: Atonal homolog 1; ASCC: Anterior 
semicircular canal; BABB: Benzyl alcohol:benzyl benzoate; BC: Blastocyst complementation; E: Embryonic day; EDTA: Ethylenediaminetetraacetic acid; ESCs: Embryonic stem cells; DAPI: 4',6-Diamidino-2-phenylindole; DPI: Dot per inch (which reflects pixels per inch); FGFR2: Fibroblast growth factor receptor 2; FIJl: Fiji Is Just ImageJ; GFP: Green fluorescent protein; hESCs: Human embryonic stem cells; HC: Hair cell; HCl: Hydrogen chloride; HCG: Human chorionic gonadotropin; hiPSCs: Human-induced pluripotent cells; HTF/ mHTF: Human tubal fluid and modified human tubal fluid; IACUC: Institute of animal care use committee; i.p.: Intraperitoneally; IPSCs: Induced pluripotent stem cell; IHC: Immunohistochemistry; LA: Lateral ampulla; LIF: Leica image file format; LSCC: Lateral semicircular canal; MHC: Major histocompatibility complex; MITF: Melanocyte inducing transcription factor; MYO6: Unconventional myosin Vl; Neurog1: Neurogenin 1; N-Myc : N-Myc proto-oncogene protein; P: Postnatal day; PA: Posterior ampulla; PBS: Phosphate-buffered saline; PBST: Phosphate-buffered saline with $0.1 \%$ Triton X-100; PCR: Polymerase chain reaction; PD: Parkinson's disease; PDX1: Pancreatic and duodenal homeobox1; PFA: Paraformaldehyde; PSCC: Posterior semicircular canal; RAR: Research animal resources; RC: Rosenthal's Canal with SGNs; SAC: Saccule; SALL1: Sal-like like protein 1; SGNs: Spiral ganglion neurons, the auditory nerve; SPF: Specific pathogenfree; SNHL: Sensorineural hearing loss; STSLIM: Scanning thin sheet laser image microscope; TFM: Tissue freezing medium; TUJ1: Class III beta-tubulin; Ut: Utricle; VG: Vestibular ganglion

\section{Acknowledgements}

We would like to acknowledge individuals from the University of Rochester, in Rochester NY, Dr. Amy Kiernan, Dr. Patricia White, Dr. J. Christopher Holt, and Dr. Lin Gan, who provided expert mentorship and training of A.S., which supported the development of this project. Additionally, we would like to acknowledge members of the Stem Cell Institute at the University of Minnesota for advice and support.

\section{Authors' contributions}

A.S. carried out the immunohistochemical studies and microscopy analysis, prepared figures, and drafted the manuscript. A. S and M.G. performed the genotyping. A.S. and M.G. harvested and processed inner ear tissue for analysis. M.G. participated in the mouse colony management and the sTSLIM analysis. Y.Y. performed the blastocyst complementation and embryo transfers. J.D. created the EGFP-labeled-induced pluripotent stem cell line. W.L. and P.S. conceived of the study. A.S., W. L, and P.S. participated in the experimental design. W.L., P.S., and A.S. provided resources. A.S., J.D., W.L., and P.S. revised the paper. The authors read and approved the final manuscript.

\section{Funding}

Research reported in this publication was supported by the National Institute on Deafness and Other Communication Disorders of the National Institutes of Health under award number F31DC015153 (Predoctoral Fellowship, ARS) and the National Institute on Aging of the National Institute of Health under award number 2T32AG029796-11 (Postdoctoral Fellowship, ARS), and a private donation from Bridget Sperl and John McCormick supported the development of the Neurog $1^{-1-}$ mice and the acquisition of resources (PAS and $\mathrm{WCL}$ ). The content is solely the responsibility of the authors and does not necessarily represent the official views of the National Institutes of Health.

\section{Availability of data and materials}

Data sharing is not applicable to this article as no datasets were generated or analyzed during the current study.

\section{Ethics approval and consent to participate}

Not applicable.

\section{Consent for publication}

Not applicable.

\section{Competing interests}

The authors declare no financial or competing interests.

\section{Author details}

'Department of Ophthalmology, University of Minnesota, Minneapolis, MN USA. ${ }^{2}$ Department of Neurosurgery, University of Minnesota, Minneapolis, MN, USA. ${ }^{3}$ Stem Cell Institute, University of Minnesota, Minneapolis, MN, USA. ${ }^{4}$ Department of Otolaryngology, University of Minnesota, Minneapolis, MN, USA. ${ }^{5}$ Mouse Genetics Laboratory, University of Minnesota, Minneapolis, MN, USA.

Received: 10 August 2020 Accepted: 24 January 2021

Published online: 12 February 2021

\section{References}

1. WHO. Deafness and hearing loss 2019. Available from: https://www.who.int/ news-room/fact-sheets/detail/deafness-and-hearing-loss.

2. Yamasoba T, Lin FR, Someya S, Kashio A, Sakamoto T, Kondo K. Current concepts in age-related hearing loss: epidemiology and mechanistic pathways. Hear Res. 2013;303:30-8.

3. Uus K, Bamford J. Effectiveness of population-based newborn hearing screening in England: ages of interventions and profile of cases. Pediatrics. 2006;117(5):e887-93.

4. Starr A, Picton TW, Sininger $Y$, Hood LJ, Berlin Cl. Auditory neuropathy. Brain. 1996:119(Pt 3):741-53.

5. Bradley J, Beale T, Graham J, Bell M. Variable long-term outcomes from cochlear implantation in children with hypoplastic auditory nerves. Cochlear Implants Int. 2008;9(1):34-60.

6. Nayagam BA, Edge AS. Stem cells for the replacement of auditory neurons. In: Dabdoub AFB, Popper AN, Fay RR, editors. The Primary Auditory Neurons of the Mammalian Cochlea. New York: Springer; 2016. p. 263-86.

7. Ito J, Kojima K, Kawaguchi S. Survival of neural stem cells in the cochlea. Acta Otolaryngol. 2001;121(2):140-2.

8. Chen W, Jongkamonwiwat N, Abbas L, Eshtan SJ, Johnson SL, Kuhn S, et al. Restoration of auditory evoked responses by human ES-cell-derived otic progenitors. Nature. 2012;490(7419):278-82.

9. Corrales CE, Pan L, Li H, Liberman MC, Heller S, Edge AS. Engraftment and differentiation of embryonic stem cell-derived neural progenitor cells in the cochlear nerve trunk: growth of processes into the organ of Corti. J Neurobiol. 2006:66(13):1489-500.

10. Kobayashi T, Yamaguchi T, Hamanaka S, Kato-Itoh M, Yamazaki Y, Ibata M, et al. Generation of rat pancreas in mouse by interspecific blastocyst injection of pluripotent stem cells. Cell. 2010;142(5):787-99.

11. Usui J, Kobayashi T, Yamaguchi T, Knisely AS, Nishinakamura R, Nakauchi H. Generation of kidney from pluripotent stem cells via blastocyst complementation. Am J Pathol. 2012;180(6):2417-26.

12. Matsunari $H$, Nagashima $H$, Watanabe M, Umeyama K, Nakano K, Nagaya $M$, et al. Blastocyst complementation generates exogenic pancreas in vivo in apancreatic cloned pigs. Proc Natl Acad Sci U S A. 2013;110(12):4557-62.

13. Goto T, Hara H, Sanbo M, Masaki H, Sato H, Yamaguchi T, et al. Generation of pluripotent stem cell-derived mouse kidneys in Sall1-targeted anephric rats. Nat Commun. 2019;10(1):451.

14. Mori M, Furuhashi K, Danielsson JA, Hirata Y, Kakiuchi M, Lin CS, et al. Generation of functional lungs via conditional blastocyst complementation using pluripotent stem cells. Nat Med. 2019;25(11):1691-8.

15. Zhang H, Huang J, Li Z, Qin G, Zhang N, Hai T, Hong Q, Zheng Q, Zhang Y, Song R, Yao J, Cao C, Zhao J, Zhou Q. Rescuing ocular development in an anophthalmic pig by blastocyst complementation. EMBO Mol Med. 2018; 10(12):e8861.

16. Crane AT, Aravalli RN, Asakura A, Grande AW, Krishna VD, Carlson DF, et al. Interspecies organogenesis for human transplantation. Cell Transplant. 2019; 28(9-10):1091-105.

17. Suchy F, Nakauchi H. Interspecies chimeras. Curr Opin Genet Dev. 2018;52: 36-41.

18. Freedman BS. Hopes and difficulties for blastocyst complementation. Nephron. 2018;138(1):42-7.

19. Zhao T, Zhang ZN, Rong Z, Xu Y. Immunogenicity of induced pluripotent stem cells. Nature. 2011;474(7350):212-5.

20. Liu X, Li W, Fu X, Xu Y. The immunogenicity and immune tolerance of pluripotent stem cell derivatives. Front Immunol. 2017;8:645.

21. Hamanaka S, Umino A, Sato H, Hayama T, Yanagida A, Mizuno N, et al. Generation of vascular endothelial cells and hematopoietic cells by blastocyst complementation. Stem Cell Reports. 2018;11(4):988-97. 
22. Najarian JS, Sutherland DE, Matas AJ, Steffes MW, Simmons RL, Goetz FC. Human islet transplantation: a preliminary report. Transplant Proc. 1977;9(1): 233-6.

23. Agarwal A, Brayman KL. Update on islet cell transplantation for type 1 diabetes. Semin Intervent Radiol. 2012;29(2):90-8.

24. Grealish S, Diguet E, Kirkeby A, Mattsson B, Heuer A, Bramoulle Y, et al. Human ESC-derived dopamine neurons show similar preclinical efficacy and potency to fetal neurons when grafted in a rat model of Parkinson's disease. Cell Stem Cell. 2014;15(5):653-65.

25. Yamaguchi T, Sato H, Kato-Itoh M, Goto T, Hara H, Sanbo M, et al. Interspecies organogenesis generates autologous functional islets. Nature. 2017;542(7640):191-6.

26. Ma Q, Chen Z, del Barco Bl, de la Pompa JL, Anderson DJ. neurogenin1 is essential for the determination of neuronal precursors for proximal cranial sensory ganglia. Neuron. 1998;20(3):469-82.

27. $\mathrm{Ma}$ Q, Anderson DJ, Fritzsch B. Neurogenin 1 null mutant ears develop fewer, morphologically normal hair cells in smaller sensory epithelia devoid of innervation. J Assoc Res Otolaryngol. 2000;1(2):129-43.

28. Steevens AR, Glatzer JC, Kellogg CC, Low WC, Santi PA, Kiernan AE. SOX2 is required for inner ear growth and cochlear nonsensory formation before sensory development. Development. 2019;146(13):dev170522. https://doi. org/10.1242/dev.170522

29. Greder LV, Gupta S, Li S, Abedin MJ, Sajini A, Segal Y, et al. Analysis of endogenous Oct4 activation during induced pluripotent stem cell reprogramming using an inducible Oct4 lineage label. Stem Cells. 2012; 30(11):2596-601.

30. Takahashi K, Yamanaka S. Induction of pluripotent stem cells from mouse embryonic and adult fibroblast cultures by defined factors. Cell. 2006;126(4): 663-76.

31. Morita S, Kojima T, Kitamura T. Plat-E: an efficient and stable system for transient packaging of retroviruses. Gene Ther. 2000;7(12):1063-6.

32. Takeo T, Nakagata N. Superovulation using the combined administration of inhibin antiserum and equine chorionic gonadotropin increases the number of ovulated oocytes in C57BL/6 female mice. PLoS One. 2015;10(5): e0128330.

33. Schroter TJ, Johnson SB, John K, Santi PA. Scanning thin-sheet laser imaging microscopy (STSLIM) with structured illumination and HiLo background rejection. Biomed Opt Express. 2012;3(1):170-7.

34. Santi PA, Johnson SB, Hillenbrand M, GrandPre PZ, Glass TJ, Leger JR. Thinsheet laser imaging microscopy for optical sectioning of thick tissues. Biotechniques. 2009;46(4):287-94.

35. Schacht $P$, Johnson SB, Santi PA. Implementation of a continuous scanning procedure and a line scan camera for thin-sheet laser imaging microscopy. Biomed Opt Express. 2010;1(2):598-609.

36. Buytaert JA, Johnson SB, Dierick M, Salih WH, Santi PA. MicroCT versus sTSLIM 3D imaging of the mouse cochlea. J Histochem Cytochem. 2013; 61(5):382-95.

37. Santi PA. Light sheet fluorescence microscopy: a review. J Histochem Cytochem. 2011:59(2):129.

38. Kopecky B, Johnson S, Schmitz H, Santi P, Fritzsch B. Scanning thin-sheet laser imaging microscopy elucidates details on mouse ear development. Dev Dyn. 2012;241(3):465-80.

39. Johnson SB, Schmitz HM, Santi PA. TSLIM imaging and a morphometric analysis of the mouse spiral ganglion. Hear Res. 2011;278(1-2):34-42.

40. Kopecky BJ, Duncan JS, Elliott KL, Fritzsch B. Three-dimensional reconstructions from optical sections of thick mouse inner ears using confocal microscopy. J Microsc. 2012;248(3):292-8.

41. Pan N, Jahan I, Kersigo J, Kopecky B, Santi P, Johnson S, et al. Conditional deletion of Atoh1 using Pax2-Cre results in viable mice without differentiated cochlear hair cells that have lost most of the organ of Corti. Hear Res. 2011;275(1-2):66-80.

42. McLaren A. Mammalian chimeras. Cambridge: Cambridge University Press; 1976.

43. Wu J, Platero-Luengo A, Sakurai M, Sugawara A, Gil MA, Yamauchi T, et al. Interspecies chimerism with mammalian pluripotent stem cells. Cell. 2017; 168(3):473-86 e15

44. Xiang AP, Mao FF, Li WQ, Park D, Ma BF, Wang T, et al. Extensive contribution of embryonic stem cells to the development of an evolutionarily divergent host. Hum Mol Genet. 2008;17(1):27-37.

45. Abello G, Khatri S, Giraldez F, Alsina B. Early regionalization of the otic placode and its regulation by the Notch signaling pathway. Mech Dev. 2007;124(7-8):631-45.
46. Raft S, Koundakjian EJ, Quinones H, Jayasena CS, Goodrich LV, Johnson JE, et al. Cross-regulation of Ngn1 and Math1 coordinates the production of neurons and sensory hair cells during inner ear development. Development. 2007:134(24):4405-15.

47. Steevens AR, Sookiasian DL, Glatzer JC, Kiernan AE. SOX2 is required for inner ear neurogenesis. Sci Rep. 2017;7(1):4086.

48. Wang X, Shi H, Zhou J, Zou Q, Zhang Q, Gou S, Chen P, Mou L, Fan N, Suo Y, Ouyang Z, Lai C, Yan Q, Lai L. Generation of rat blood vasculature and hematopoietic cells in rat-mouse chimeras by blastocyst complementation. J Genet Genomics. 2020;47(5):249-61.

49. Theunissen TW, Friedli M, He Y, Planet E, O'Neil RC, Markoulaki S, et al. Molecular criteria for defining the naive human pluripotent state. Cell Stem Cell. 2016;19(4):502-15.

50. Gafni O, Weinberger L, Mansour AA, Manor YS, Chomsky E, Ben-Yosef D, et al. Derivation of novel human ground state naive pluripotent stem cells. Nature. 2013;504(7479):282-6.

51. Wang X, Li T, Cui T, Yu D, Liu C, Jiang L, et al. Human embryonic stem cells contribute to embryonic and extraembryonic lineages in mouse embryos upon inhibition of apoptosis. Cell Res. 2018;28(1):126-9.

52. Huang K, Zhu Y, Ma Y, Zhao B, Fan N, Li Y, et al. BMl1 enables interspecies chimerism with human pluripotent stem cells. Nat Commun. 2018;9(1):4649.

53. Masaki H, Kato-Itoh M, Takahashi Y, Umino A, Sato H, Ito K, et al. Inhibition of apoptosis overcomes stage-related compatibility barriers to chimera formation in mouse embryos. Cell Stem Cell. 2016;19(5):587-92.

54. Wu J, Okamura D, Li M, Suzuki K, Luo C, Ma L, et al. An alternative pluripotent state confers interspecies chimaeric competency. Nature. 2015; 521(7552):316-21.

55. James D, Noggle SA, Swigut T, Brivanlou AH. Contribution of human embryonic stem cells to mouse blastocysts. Dev Biol. 2006;295(1):90-102.

56. Mascetti VL, Pedersen RA. Human-mouse chimerism validates human stem cell pluripotency. Cell Stem Cell. 2016;18(1):67-72.

57. de Almeida PE, Meyer EH, Kooreman NG, Diecke S, Dey D, Sanchez-Freire V, et al. Transplanted terminally differentiated induced pluripotent stem cells are accepted by immune mechanisms similar to self-tolerance. Nat Commun. 2014;5:3903.

58. Guha P, Morgan JW, Mostoslavsky G, Rodrigues NP, Boyd AS. Lack of immune response to differentiated cells derived from syngeneic induced pluripotent stem cells. Cell Stem Cell. 2013;12(4):407-12.

59. Yoshihara M, Hayashizaki Y, Murakawa Y. Genomic instability of iPSCs: challenges towards their clinical applications. Stem Cell Rev Rep. 2017;13(1): 7-16.

60. Kruse V, Hamann C, Monecke S, Cyganek L, Elsner L, Hubscher D, et al. Human induced pluripotent stem cells are targets for allogeneic and autologous natural killer (NK) cells and killing is partly mediated by the activating NK receptor DNAM-1. PLoS One. 2015;10(5):e0125544.

61. Benichou G, Gonzalez B, Marino J, Ayasoufi K, Valujskikh A. Role of memory T cells in allograft rejection and tolerance. Front Immunol. 2017;8:170.

62. Rong Z, Wang M, Hu Z, Stradner M, Zhu S, Kong H, et al. An effective approach to prevent immune rejection of human ESC-derived allografts. Cell Stem Cell. 2014;14(1):121-30.

63. Szot GL, Yadav M, Lang J, Kroon E, Kerr J, Kadoya K, et al. Tolerance induction and reversal of diabetes in mice transplanted with human embryonic stem cell-derived pancreatic endoderm. Cell Stem Cell. 2015; 16(2):148-57.

64. Mann ZF, Gálvez H, Pedreno D, et al. Shaping of inner ear sensory organs through antagonistic interactions between Notch signalling and Lmx1a. Elife. 2017:6:e33323. Published 2017 Dec 4. https://doi.org/10.7554/ elife.33323.

65. Park IH, Zhao R, West JA, Yabuuchi A, Huo H, Ince TA, et al. Reprogramming of human somatic cells to pluripotency with defined factors. Nature. 2008; 451(7175):141-6.

\section{Publisher's Note}

Springer Nature remains neutral with regard to jurisdictional claims in published maps and institutional affiliations. 